Relations industrielles

Industrial Relations

\title{
Jacques de MONLÉON : Marx et Aristote. Perspectives sur l'homme. Paris, FAC éditions, 1984, ISBN 0317-0179
}

\section{Maurice Lebel}

Volume 41, numéro 4, 1986

URI : https://id.erudit.org/iderudit/050274ar

DOI : https://doi.org/10.7202/050274ar

Aller au sommaire du numéro

Éditeur(s)

Département des relations industrielles de l'Université Laval

ISSN

0034-379X (imprimé)

1703-8138 (numérique)

Découvrir la revue

Citer ce compte rendu

Lebel, M. (1986). Compte rendu de [Jacques de MONLÉON : Marx et Aristote. Perspectives sur l'homme. Paris, FAC éditions, 1984, ISBN 0317-0179]. Relations industrielles / Industrial Relations, 41(4), 883-884.

https://doi.org/10.7202/050274ar

Tous droits réservés @ C Département des relations industrielles de l'Université Laval, 1986
Ce document est protégé par la loi sur le droit d'auteur. L’utilisation des services d'Érudit (y compris la reproduction) est assujettie à sa politique d'utilisation que vous pouvez consulter en ligne.

https://apropos.erudit.org/fr/usagers/politique-dutilisation/ 
Marx et Aristote. Perspectives sur l'homme, par Jacques de Monléon, Paris, FAC éditions, 30, rue Madame, 1984, ISBN 0317-0179

Cet ouvrage posthume de Jacques de Monléon (1901-1981) fait penser par le rapprochement limpide et surprenant qu'il établit entre Marx et Aristote. L'auteur, ancien professeur de philosophie pendant un demi-siècle tantôt à l'Institut Catholique de Paris et à l'Université Laval à Québec, tantôt à l'Université Libre des Sciences de l'Homme à Paris, invite le lecteur à étudier Le Capital de Karl Marx et La Politique d'Aristote, car ce sont deux livres majeurs, fondamentaux, difficiles à lire, il est vrai, mais indispensables pour comprendre le monde d'aujourd'hui. Aristote a beau avoir écrit 400 ans avant Jésus-Christ et Karl Marx au XIX ${ }^{e}$ siècle, ils ne sont pas moins pour autant nos contemporains par les idées qu'ils ont exprimées chacun dans son volume respectif; ils gagnent même beaucoup à être confrontés, le premier ayant écrit sur l'homme et la cité politique avant la Révélation, le second ayant traité de l'homme et de la société économique comme si la Révélation n'avait jamais eu lieu. Pour comprendre Marx, il faut remonter à Aristote et à saint Thomas d'Aquin.

Ce qui frappe dans ce livre, composé de six leçons, dont quatre sont consacrées à l'idéalisme et à la praxis selon Marx, deux à La Politique d'Aristote, notamment à la Cité et aux étapes du développement social, c'est la densité de l'expression, la rigueur de l'argumentation et la dialectique serrée. Autant dire qu'on doit en faire une lecture lente et réfléchie. Ce qu'on remarque aussi, c'est l'abondante documentation: on y voit défiler, pour ainsi dire, plus d'une vingtaine d'historiens, d'économistes et de philosophes, de Thucydide et Platon à Durkheim et à Adriano Tilgher. Sans oublier James I. Stewart (1712-1780) dont les idées économiques, philosophiques et politiques étaient familières à Marx, fait que ce dernier ne reconnaît pas trop souvent. De plus, Jacques de Monléon admet honnêtement, voire avec une évidente sympathie, que Marx s'accorde avec Aristote sur plusieurs points. Mais il vise plutôt à mettre le lecteur en garde contre les «séduisantes» théories marxistes.

Elles le sont, en effet, à longueur de journée: on les lit, on les entend et on les emploie à son insu. Elles sont devenues monnaie courante, le primat de l'homo faber, de l'économique tendant à l'emporter aujourd'hui sur tout le reste dans notre civilisation qui est surtout, mais pas exclusivement, industrielle et technicienne; on parle même tout bonnement, sans trop réfléchir, encore moins sans sourciller ou rager de colère, des industries culturelles, de l'industrie du tourisme, comme on parle de démocratie populaire (quelle tautologie!), de création ou de créativité, d'atelier, de techniques: alimentaires, artistiques, électorales, littéraires, médicales, syndicales, vestimentaires, et j'en passe. On ne parle que de production et de surproduction, sauf des enfants, bien sûr; mais ce qui manque le plus dans notre monde, ce sont les enfants, à commencer par le Québec, où la dénatalité est un véritable suicide collectif et volontaire. En bref, l'important est de produire. Quoi? Comment? Dans quelles conditions? Pour qui? Pour quoi? N'importe. Artistes et écrivains, industriels et scientifiques parlent de leurs productions comme s'ils ne faisaient rien d'autre dans leur existence. En d'autres termes, c'est le travail pour le travail. La religion du travail.

J'aurais des loisirs, je composerais un essai pour établir que Le Capital, loin d'être une célèbre apologie du travail, avilit plutôt le travail au lieu de l'ennoblir, car le grand tort de Marx est de tenir l'homme essentiellement, uniquement pour un animal capable de produire ses propres moyens d'existence, comme si la contemplation, l'observation, l'étude, l'émerveillement, la poésie, la soif du bonheur et du savoir, de l'absolu, de l'immortalité et de la perfection n'existaient point en lui; comme si l'homme n'était point, comme l'a défini Platon, «une plante céleste», comme s'il n'était point, selon Aristote, un animal politique et social, vivant en communauté et doué d'intelligence et de volonté libre. Pour Aristote, qui part de l'observation de la réalité humaine, le savoir, l'agir et le faire en constituent la hiérarchie des valeurs. Pour le 
philosophe allemand de l'économie politique, c'est le faire qui distingue essentiellement l'homme des autres animaux. Pour le philosophe grec, c'est la pensée, dont l'agir et le faire sont des modalités, c'est elle qui est le fondement même de l'acte humain, libre et responsable, car il n'y a pas de liberté sans responsabilité. C'est à ce titre d'homme pensant, parlant, de l'homo loquens, qu'il échappe au déterminisme animal, minéral et végétal.

Dans la cité démocratique, l'homme atteint son plein développement, sa perfection. Il y passe du statut de l'homo faber - ce qu'il était primitivement - à celui de l'homo loquens, du domaine naturel de la consanguinité, de la famille, de la tribu, à celui, plus global et plus rationnel, de la cité, de l'amitié et de la justice. Tel est le point de vue d'Aristote, qui tient l'amitié et la justice pour les deux sommets de la vie morale dans la société politique, étant donné que l'intelligence et la volonté libre sont des principes premiers pour l'homme. En revanche, ce qui distingue l'homme, pour Marx, ce n'est ni l'intelligence ni la volonté libre, mais la capacité corporelle ou physique de fabriquer ses moyens d'existence; dans le matérialisme marxiste, la pensée ne joue pas le premier rôle, et la praxis, si chère aux Grecs, devient le faire pour Marx, tandis qu'elle est l'acte humain pour Aristote; dans l'esprit de Marx, la pensée se dégage de l'action productrice au lieu de la précéder; il s'ensuit que beaucoup de gens finissent par penser comme ils vivent au lieu de faire le contraire. De là sa conception du travail: une dépense de force humaine. C'est tout. Il n'y a rien d'autre. Comme on est loin de Virgile qui concevait le travail comme facteur suprême de la valeur de l'homme! Ainsi est bouclé le cercle de plomb. Décidément, le communisme devrait s'appeler le terréisme. Et avec Voltaire de s'écrier:

«Qu'il fait bon de vivre dans ce siècle de fer!»

Marx et Aristote, on le voit, ouvre des perspectives profondes et vraies sur l'homme, qu'il ne faut jamais perdre de vue. Notre malheur vient du fait que nous l'oublions trop souvent.

Maurice LEBEL

Université Laval

Organization Theory: A Macro Perspective for Managing, by J.H. Jackson, C.P. Morgan and J.G.P. Prolillo, Englewood Cliffs, New Jersey, Prentice-Hall, 1986, 387 pp., ISBN 0-13-641572-5-01

Organization Theory: Integrating Structure and Behavior, by Gary Dessler, Englewood Cliffs, New Jersey, Prentice-Hall, 1986, 477 pp., ISBN 0-13-641-903-8-01

These are two updated manuals on organization theory. That by Jackson et al, was reviewed here in its first edition. The latest edition - the third - is distinguished from its predecessors by putting the focus «on the macro aspects of organizations, utilizing both conceptual and empirical studies in organization theory" (xi). It includes chapters on: Organizations; Organizational effectiveness and goals, the Environment and interorganizational relations; Bureaucracy and Structure; Organizational design; Patterns of business organization design; Organizing size; Technology and its impact on the organization; Intraorganizational issues (power, control, rules); and Organizational survival, decline, change and growth. Each chapter is broken down into a series of logical subdivisions, followed by a summary, suggestions for further reading, and a section including integral readings on the subject treated by the chapter.

In the case of Dessler's book, this is a second edition. According to the author it involves several improvements over its predecessor: 